tourismuspolitische Geschäfte begutachtet. Der Kommission gehören je zwei Vertreter aus den Bereichen Forschung, Politik und Praxis an. Zudem sind die beiden öffentlich-rechtlichen Körperschaften des Bundes im Bereiche des Tourismus, die Schweizerische Verkehrszentrale und die Schweizerische Gesellschaft für Hotelkredit sowie fünf eidgenössische Departemente in der Kommission vertreten.

\section{Der Fremdenverkehrsdienst als Fachstelle fuir Tourismus der Bundesverwaltung}

Auf Verwaltungsebene besteht mit dem Fremdenverkehrsdienst des Bundesamtes für Verkehr eine Fachstelle für Tourismus, die grundsätzlich alle den Tourismus betreffenden Angelegenheiten bearbeitet. Ausnahme bilden die ausdrücklich durch Gesetz und Verordnung einer anderen Stelle zugeordneten Aufgaben (z. B. Fremdenverkehrsstatistik). Der Fremdenverkehrsdienst setzt sich für günstige Rahmenbedingungen und eine geordnete Entwicklung des Tourismus als bedeutende Freizeitform und Wirtschaftsbranche ein. Obwohl personell bescheiden dotiert, versucht er, die Anliegen des Schweizer Tourismuskonzeptes in die Praxis umzusetzen. Der Bundesrat hat dazu mit der Verbindlicherklärung des Konzeptes für die Verwaltung die Voraussetzungen geschaffen. Als wesentliches Instrument für die Durchsetzung der Tourismuspolitik kann der Fremdenverkehrsdienst die Konzessionspolitik für touristische Transportanlagen einsetzen. Im Bereiche des touristischen Marketings beschäftigt sich der Dienst mit der Aufsicht und der Finanzierung der Landeswerbung der SVZ und unterstützt die Informations- und Beratungstätigkeit des Schweizerischen Fremdenverkehrsverbandes zugunsten der landesweiten Angebotsgestaltung. Zudem befaßt er sich mit zahlreichen tourismuspolitischen Sondergeschäften, die von der Aufsicht über ein Feriendorf für Jugendliche bis zur Bearbeitung von Beitragsgesuchen für die Durchführung Olympischer Spiele reichen. Der Fremdenverkehrsdienst vertritt die Schweiz auch in den intergouvernementalen Organisationen, beispielsweise in der Weltorganisation für Tourismus oder im Tourismuskomitee der OECD, und arbeitet bilateral mit den Tourismusbehörden anderer Reiseländer zusammen.

Ein besonderes Anliegen der Fachstelle ist die Koordination der touristischen Anliegen auf Bundesebene und die Zusammenarbeit mit den Kantonen und Trägern des Tourismus. Der Fremdenverkehrsdienst führt das Sekretariat der beratenden Kommission für Fremdenverkehr. Er arbeitet in den Organen nationaler touristischer Institutionen mit. Tourismus ist eine Querschnittaufgabe, die in viele Lebensbereiche hineingreift. Bei der komplexen und dezentralisierten Organisation des schweizerischen Tourismus braucht es stetige Bemühungen, da $\beta$ der Blick auf das touristische Ganze nicht verlorengeht. Zusammen mit unseren Partnern bei Behörden und Privaten setzen wir uns dafür ein. Ab 1984 wird der Fremdenverkehrsdienst dem Bundesamt für Industrie, Gewerbe und Arbeit im Volkswirtschaftsdepartement angegliedert.

Fremdenverkehrsdienst, Bundesamt für Verkehr, Bundeshaus Nord, CH-3003 Bern, Tel. 031/6157 94/92

Leiter: Dr. Peter Keller, Stv.: lic. rer. pol. Peter Schmid

\title{
Die Sektion Tourismus im Bundesamt für Statistik
}

Das Bundesamt für Statistik (BFS) wurde 1933 beauftragt, eine «regelmäßige Statistik der in den Beherbergungsstätten abgestiegenen Gäste» durchzuführen. Die Betreuung der fremdenverkehrsstatistischen Erhebungen obliegt der Sektion Tourismus, die 26 Mitarbeiterinnen und Mitarbeiter beschäftigt.

Die gesetzlichen Grundlagen zur eidgenössischen Fremdenverkehrsstatistik aus dem Jahre 1933 wurden vorerst nur teilweise ausgenützt; sie verlangten vorderhand lediglich die Erfassung der in den Hotel- und Kurbetrieben abgestiegenen Gäste. Heute werden diese Grunddaten mittels offizieller Formulare direkt bei den Inhabern und Leitern der rund 8000 meldepflichtigen Hotels, Pensionen, Gasthäuser, Motelș, Bäder- kliniken, Volksheilbäder, Kurhäuser und Höhensanatorien eingeholt. Ankünfte und Logiernächte aufgeteilt nach Wohnsitzländern der Gäste - werden dabei monatlich registriert, ebenso das Bettenangebot, die Öffnungszeiten der Betriebe und die Zahl der beschäftigten Personen. Jeweils am 25. des dem Berichtsmonat folgenden Monats sind die ersten gesamtschweizerischen Ergebnisse verfügbar, die in Form einer Pressemitteilung verbreitet werden. Die DetailÜbersichten erscheinen etwa zehn Tage später. Publiziert und kommentiert werden Monats-, Saisonund Jahresergebnisse, geordnet nach touristischen Regionen, Zonen, Fremdenverkehrsorten, Städten, Herkunftsländern, Preisklassen, Betriebsarten usw. 
Die Statistik der Parahotellerie erwies sich im Laufe der sechziger Jahre als eine Notwendigkeit, um die atemraubende Nachfrageentwicklung auf diesem Sektor zahlenmäßig verfolgen zu können. Das Eidgenössische Departement des Innern verlangte daher im Jahre 1965 vom Bundesamt für Statistik (BFS) die Ausdehnung der Erhebungen auf die wichtigsten Beherbergungsformen der Parahotellerie und erließ am 16. November 1978 die "Verordnung über die Fremdenverkehrsstatistik in der Parahotellerie», welche die zu erfassenden Beherbergungsformen eindeutig umschreibt. Aus finanziellen und personellen Gründen erfolgte der Aufbau der ParahotellerieStatistik nur schrittweise. Unvollständig ist die Erfassung heute nur noch im Teilbereich "Chalets und Ferienwohnungen", wogegen die Gäste in den übrigen Beherbergungsarten «vollständig» erfaßt werden.

Die schweizerische Statistik der Parahotellerie umfaßt heute:

\section{Chalets, Ferienwohnungen, Privatzimmer}

Die Datenerfassung erfolgt über Verkehrsvereine und Gemeinden und ist mit dem Einzug der Kurtaxen oder der Polizeimeldescheine gekoppelt. Die vollständige Erfassung der in Privatquartieren abgestiegenen Kundschaft erstreckt sich auf die Kantone Bern, Obwalden, Appenzell A.R., St.Gallen, Graubünden, Tessin, Wallis und Waadt sowie auf einige Fremdenverkehrsorte in anderen Kantonen.

\section{Campingplätze, Gruppenunterkünfte und}

Jugendherbergen (SJH)

Vorderhand werden gesamtschweizerische Ergebnisse der Parahotellerie nur jährlich publiziert. In dieser
Publikation sowie den alljährlich erscheinenden Berichten über jene Kantone, in welchen die Gäste aller Beherbergungsformen vollständig erfaßt werden, sind die Daten gegliedert nach Unterkunftsformen, Herkunftsländern, Winter- und Sommerhalbjahr und nach Fremdenverkehrsorten.

Die Arbeiten über die volkswirtschaftliche Bedeutung des Tourismus konzentrieren sich auf die Erstellung der Fremdenverkehrsbilanz, also auf die Ermittlung von Einnahmen und Ausgaben im Fremdenverkehr mit dem Ausland. Die Fremdenverkehrsbilanz umfaßt nach internationalen Konventionen folgende Hauptpositionen:

- Ferien- und Geschäftsaufenthalte;

- Kur-, Spital- und Studienaufenthalte;

- Tages- und Transitverkehr;

- internationale Personentransporte.

Vielfältig sind die übrigen Aufgaben der Sektion Tourismus. Sie reichen von den zahlreichen, vielfach mit arbeitsintensiven Sonderauszählungen und $\mathrm{Zu}$ sammenstellungen verbundenen Auskunftserteilungen über die Zusammenarbeit mit internationalen Gremien (OECD in Paris, Weltorganisation für Tourismus in Madrid usw.), Sonderaufgaben in Zusammenhang mit der Berechnung unseres Landesindexes der Konsumentenpreise bis zur Ermittlung der Frequenzen von ins Ausland gereisten Schweizern.

\section{Heinz Kleine}

Bundesamt für Statistik (BFS), Sektion Tourismus

Hallwylstr. 15, 3003 Bern, Tel. 031/61 8665

\section{Die Schweizerische Verkehrszentrale (SVZ)}

Vor dem Hintergrund der zunehmenden Bedeutung des Tourismus in unserem Land regte Nationalrat A.Seiler 1911 in einer Motion die Schaffung einer «Zentralstelle behufs Förderung des Fremdenverkehrs in der Schweiz» an. Mit der Gründung der «Nationalen Vereinigung zur Förderung des Reiseverkehrs» wurde der Vorschlag 1917 in die Tat umgesetzt. Ein Jahr später errichtete die neue, rein privatrechtlich strukturierte Organisation ihren Geschäftssitz unter der Bezeichnung «Schweizerische Verkehrszentrale (SVZ)" in Zürich. Wie schon zuvor betrieb man die Landeswerbung im Ausland über die Agenturen der Schweizerischen Bundesbahnen (SBB) in Paris, New York, Berlin, Kairo und Wien. Eine Ausnahme stellte lediglich Nizza dar, wo die SVZ 1923 ein eigenes Büro eröffnete. Als im Gefolge der Weltwirtschaftskrise auch die Logierfrequenzen in der Schweiz rapide sanken, drängte sich auf dem Gebiet der Auslandwerbung eine engere Zusammenarbeit der vorhandenen Verkehrsorganisationen auf.

1933 führten diese Bestrebungen zwischen den SBB, der SVZ und der PTT-Verwaltung zum Abschluß einer "Werbegemeinschaft», zu der sich wenig später auch noch das Eidgenössische Amt für Verkehr gesellte. Die SVZ war für die Bereitstellung des Propagandamaterials zuständig und erhielt das Recht, eigene Mitarbeiter in die SBB-Vertretungen zu entsenden, deren Zahl in den folgenden Jahren auf elf 